\title{
Quantitative Trait Locus Mapping for Common Scab Resistance in a Tetraploid Potato Full-Sib Population
}

\author{
Guilherme da Silva Pereira, ${ }^{1, \dagger}$ Marcelo Mollinari, ${ }^{2,3}$ Xinshun Qu, ${ }^{4}$ Christian Thill, ${ }^{5}$ Zhao-Bang Zeng, ${ }^{2,3}$ Kathleen Haynes, ${ }^{6}$ and \\ G. Craig Yencho \\ ${ }^{1}$ International Potato Center, ILRI Campus, Nairobi 25171-00603, Kenya \\ ${ }^{2}$ Department of Horticultural Science, North Carolina State University, Raleigh, NC 27695, U.S.A. \\ ${ }^{3}$ Bioinformatics Research Center, North Carolina State University, Raleigh, NC 27695, U.S.A. \\ ${ }^{4}$ Department of Plant Pathology and Environmental Microbiology, The Pennsylvania State University, University Park, PA 16802, U.S.A. \\ ${ }^{5}$ Department of Horticultural Science, University of Minnesota, St. Paul, MN 55108, U.S.A. \\ ${ }^{6}$ Genetic Improvement of Fruits and Vegetables Laboratory, U.S. Department of Agriculture-Agricultural Research Service, Beltsville, MD \\ 20705, U.S.A.
}

\begin{abstract}
Despite the negative impact of common scab (Streptomyces spp.) on the potato industry, little is known about the genetic architecture of resistance to this bacterial disease in the crop. We evaluated a mapping population ( $\sim 150$ full sibs) derived from a cross between two tetraploid potatoes ('Atlantic' $\times$ B1829-5) in three environments (MN11, PA11, ME12) under natural common scab pressure. Three measures to common scab reaction, namely percentage of scabby tubers and disease area and lesion indices, were found to be highly correlated $(>0.76)$. Because of the large environmental effect, heritability values were

zero for all three traits in MN11, but moderate to high in PA11 and ME12 ( $\sim .44$ to 0.79$)$. We identified a single quantitative trait locus (QTL) for lesion index in PA11, ME12, and joint analyses on linkage group 3, explaining $\sim 22$ to $30 \%$ of the total variation. The identification of QTL haplotypes and candidate genes contributing to disease resistance can support genomics-assisted breeding approaches in the crop.

Keywords: disease resistance, polyploid QTL model, single-nucleotide polymorphism, Solanum tuberosum, Streptomyces spp.
\end{abstract}

Common scab of potato (Solanum tuberosum L.) is an economically important disease that occurs worldwide. It is caused by pathogenic soilborne bacteria belonging to several species in the genus Streptomyces (Loria et al. 1995; Wanner 2009). Common scab is characterized by brownish superficial, raised, or pitted lesions on tuber surfaces, where the phytotoxin Thaxtomin A produced by pathogenic Streptomyces spp. plays an important role (Kinkel et al. 1998; Loria et al. 1995). This disease is highly influenced by the environment (Haynes et al. 2010), especially by the soil conditions (Krištůfek et al. 2015), and by the virulence of the pathogens present in the soil (Clarke et al. 2019; Wanner 2006; Wanner and Haynes 2009).

Although some management practices have been proposed to mitigate common scab damages (Dees and Wanner 2012), there are currently no chemical or cultural management approaches that provide effective control of the disease. Breeding for varietal resistance is still being pursued as a more desirable solution (Braun et al. 2017b; Navarro et al. 2015). However, the development of resistant varieties, as well as the study of the genetic architecture of resistance in potato, is quite challenging. Despite scab symptoms exhibiting a

\section{${ }^{\dagger}$ Corresponding author: G. da Silva Pereira; g.pereira@ufv.br}

Current address of G. da Silva Pereira: Department of Agronomy, Federal University of Viçosa, Viçosa 36570-900, Brazil.

\section{Thill is deceased.}

Funding: The work of G. da Silva Pereira and M. Mollinari on QTLpoly and MAPpoly has been funded by Bill and Melinda Gates Foundation grant nos. OPP1052983 and OPP1213329.

*The $\boldsymbol{e}$-Xtra logo stands for "electronic extra" and indicates supplementary materials are published online.

The author(s) declare no conflict of interest.

Accepted for publication 9 March 2021.

Copyright $\odot 2021$ The Author(s). This is an open access article distributed under the CC BY 4.0 International license. quantitative distribution (Haynes et al. 1997, 2009), scab resistance has been postulated as having an oligogenic nature, with a dominant and a recessive locus (Murphy et al. 1995). Because of the autotetraploidy of potato $(2 n=4 x=48)$, such a recessive locus would have to appear in a quadruplex recessive state, which is not easy to achieve using conventional breeding (Bradshaw 2017). In this sense, marker-assisted selection (MAS) could facilitate recessive allele introgression (Bethke et al. 2019).

Developments in genomics and bioinformatics tools have allowed several technical difficulties to be overcome when studying the genetic architecture of a trait in autopolyploid crops. For potato, strategies based on genotyping by sequencing (Sverrisdóttir et al. 2017; Uitdewilligen et al. 2013) or chip arrays (Felcher et al. 2012; Vos et al. 2015) can now provide allele intensity information of thousands of single nucleotide polymorphisms (SNPs). After dosage calling is carried out (Schmitz Carley et al. 2017; Zych et al. 2019), these variants can be ultimately utilized in several applications such as linkage and quantitative trait locus (QTL) analyses (Chen et al. 2018; da Silva Pereira et al. 2020b; Hackett et al. 2014), genome-wide association studies (GWAS) (Rosyara et al. 2016; Yuan et al. 2019), or genomic-assisted prediction (EncisoRodriguez et al. 2018; Sverrisdóttir et al. 2017).

In the case of QTL identification of resistance to common scab in potato, only a few studies have been carried out so far. The first one involved a tetraploid '12601ab1' $\times$ 'Stirling' mapping population $(N$ $=227 \mathrm{~F}_{1}$ clones), and the discovery of QTL on homology groups II and IV (Bradshaw et al. 2008). Later, a diploid 'US-W4' $\times$ '524-8' mapping population ( $N=\sim 49$ to $91 \mathrm{~F}_{2}$ clones) facilitated the identification of a single QTL on chromosome 11 (Braun et al. 2017a). Another tetraploid mapping population $\left(N=151 \mathrm{~F}_{1}\right.$ clones) derived from the cross 'Atlantic' $\times$ 'Superior' was used to detect QTL on chromosomes $1,2,3,5,6,10$, and 11 (Zorrilla et al. 2021). In addition, GWAS was performed in a tetraploid diversity panel ( $N=143$ clones $)$ with significant associations on chromosomes 2, 4, and 12 (Yuan et al. 2019), and in a 'Tundra' $\times$ 'Kalkaska' full-sib population $\left(N=198 \mathrm{~F}_{1}\right.$ clones), which identified QTL on chromosomes 1 and 2 (Kaiser et al. 2020).

In order to expand the understanding of the genetic control of common scab resistance in tetraploid potatoes, we evaluated a full-sib population with $\sim 150$ clones in three environments where common scab is of natural occurrence. An integrated genetic map based on a dosage- 
sensitive SNP chip array (da Silva Pereira et al. 2020b) was used for QTL mapping and helped us to estimate haplotype-specific additive effects and to pinpoint candidate genes in the $S$. tuberosum genome that could potentially help breeders in deploying MAS for common scab in potato.

\section{Materials and Methods}

$\mathbf{F}_{1}$ population and field trials. A mapping population named B2721, initially composed of 156 full sibs, was derived from a cross between 'Atlantic' and B1829-5, and it was previously analyzed regarding its segregation to internal heat necrosis and several yieldrelated and quality-related traits (da Silva Pereira et al. 2020b; McCord et al. 2011; Schumann et al. 2017). 'Atlantic' is a widely grown chipping variety in the United States, whereas B1829-5 is an advanced round white clone from the U.S. Department of AgricultureAgricultural Research Service Beltsville potato breeding program. Although both parents have shown susceptibility to common scab, B1829-5 was found to be less susceptible than 'Atlantic'.

The B2721 was evaluated at three locations (hereafter also referred to as environments) in Becker, Minnesota, in 2011 (MN11), in Pennsylvania Furnace, Pennsylvania, in 2011 (PA11), and in Presque Isle, Maine, in 2012 (ME12) in fields with a history of common scab pressure. In each location, the experimental design consisted of a randomized complete block with two replications, with four hills per plot. A total of 153 full sibs were evaluated across locations, where MN11 included 146 full sibs plus one check (B1829-5), PA11 included 151 full sibs plus two checks ('Atlantic' and B1829-5), and ME12 included 139 full sibs plus six checks (B1829-5, 'Atlantic' and 'Green Mountain' as relatively susceptible, and 'Ontario', 'Russet Burbank', and 'Superior' as relatively resistant clones). All trials were carried out from early June (7 to 12) to late September (19 to 26) of their respective years. Standard crop management practices for the respective locations were followed.

All tubers were collected and visually assessed per plot for three traits of interest. First, the percentage of tubers with scab lesions in each plot (PS) was calculated. Second, the tubers were rated for percentage of surface area covered by lesions $(0=$ no scab; $1=0.1$ to $3 \%$ surface area; $2=3.1$ to $6 \% ; 3=6.1$ to $12 \% ; 4=12.1$ to $25 \%$; $5=25.1$ to $50 \% ; 6=>50 \%$ ) following modified Horsfall-Barratt scale (Horsfall and Barratt 1945), which was then converted to an area index (AI) as the sum of the individual tuber ratings of surface area infected divided by six times the number of tubers (Goth et al. 1993). Third, the tubers were also rated for type of lesion $(0=$ no lesions; $1=$ superficial discrete; 2 = coalescing superficial; 3 = raised discrete; 4 = raised coalescing; 5 = pitted discrete and coalescing) following James (1971), which was then converted to a lesion index (LI) as the sum of the individual tuber ratings of lesion type divided by five times the number of tubers (Goth et al. 1993). The average number of tubers scored per plot was 30 in MN11, 24 in PA11, and 21 in ME12.

Phenotypic analyses. Adjusted means were obtained based on a two-stage analysis approach using ASReml-R package v. 4.1.0 (Butler et al. 2018) and its restricted maximum likelihood (REML) estimation algorithm. In the first stage, phenotypic data were fitted for each separate environment using the model $y_{i j}=\mu+b_{j}+g_{i}+\varepsilon_{i j}$, where $y_{i j}$ is the phenotypic value of individual $i$ in block $j, \mu$ is the intercept, $b_{j}$ is the fixed effect of block $j(j=1, \ldots, J ; J=2), g_{i}$ is the fixed effect of individual $i\left(i=1, \ldots, n ; n=n_{g}+n_{c}\right.$ with $n_{\mathrm{g}}=139,146$, or 151 full sibs, and $n_{\mathrm{c}}=1,2$, or 6 checks depending on the environment), and $\varepsilon_{i j}$ is the random effect of the residual error with $\varepsilon_{i j} \sim N\left(0, \sigma^{2}\right)$.

In the second stage, both adjusted means and weights derived from the diagonal of the variance-covariance inverse matrix from each firststage model (method 4 of Möhring and Piepho 2009) were used to fit the model $\mu_{i k}=\phi+g_{i}+e_{k}+g e_{i k}+\varepsilon_{i k}$, where $\mu_{i k}$ is the adjusted mean of individual $i$ in environment $k$ from the first-stage model, $\phi$ is the intercept, $g_{i}$ is the fixed effect of individual $i(i=1, \ldots, n), e_{k}$ is the random effect of environment $k(k=1, \ldots, K ; K=3)$ with $e_{k} \sim N\left(0, \sigma_{e}^{2}\right), g e_{i k}$ is the random effect of genotype-by-environment interaction with $g e_{i k} \sim N\left(0, \sigma_{g e}^{2}\right)$, and $\varepsilon_{i k}$ is the random effect of the residual error as a function of the weights from the first-stage model.

Approximate broad-sense heritability values were calculated using $H^{2}=1-P E V / \sigma_{G}^{2}$ (Cullis et al. 2006; Isik et al. 2017, p. 223), where
$P E V$ is the best linear unbiased prediction error variance, and $\sigma_{G}^{2}$ is the genetic variance associated with $g_{i}$ when full-sib genotypes were treated as random in the two previous models, i.e., $g_{i} \sim N\left(0, \sigma_{G}^{2}\right)$. Pearson's correlations were estimated between pairs of adjusted means derived from the first- stage and second-stage models using Hmisc R package v. 4.3-0 (Harrel 2019), correlograms were plotted using ggcorrplot2 R package v. 0.1.0 (Cai 2019), and the remaining plots were obtained using ggplot2 R package v. 3.3.2 (Wickham 2016).

QTL mapping analyses. An integrated, fully phased genetic map was constructed using MAPpoly R package v. 0.1.0 (Mollinari et al. $2020)$ for the same $\mathrm{B} 2721$ population $\left(N=156 \mathrm{~F}_{1}\right.$ clones) by Pereira et al. (2020b). This map is 1,630-centimorgan (cM) long, comprises the $12 \mathrm{~S}$. tuberosum base chromosomes, and contains 4,285 SNPs derived from the Illumina Infinium 8,303 Potato Array (Felcher et al. 2012). All details about the linkage map construction can be found at da Silva Pereira et al. (2020b). Genotype conditional probabilities were computed every centimorgan using a hidden Markov model adapted to autopolyploids (Mollinari and Garcia 2019) and, ultimately, employed in the QTL analyses.

For each trait, we used a random-effect multiple interval mapping (REMIM) model implemented in QTLpoly R package v. 0.2.1 (da Silva Pereira et al. 2020a) for QTL detection using the adjusted means derived from the phenotypic analyses. In such a model, variance components associated with putative QTL, treated as random effects, were tested using score statistics. $P$ values were compared with a genomewide significance $(\alpha)$ assessed via score-based resampling method (Zou et al. 2004). In short, QTL were added (forward search) to a random-effect model using a more relaxed genome-wide significance level $(\alpha=0.20)$. Then, QTL already in the model were reevaluated under a more stringent significance level $(\alpha=0.05)$ and excluded (backward elimination) if not significant. These steps were repeated under the more stringent significance level $(\alpha=0.05)$, and the forward-backward algorithm was stopped once no more QTL were either added to or excluded from the model. Putative QTL were tested every centimorgan position along the B2721 genetic map. Additionally, a 20-cM window on each side of QTL already in the model was used to avoid two QTL in very close positions. The variance component estimates were used to compute the QTL heritability, $h_{\mathrm{QTL}}^{2}$, as the ratio between the additive variance of a QTL, $\sigma_{\mathrm{QTL}}^{2}$, and the total phenotypic variance, $\sigma_{P}^{2}$ (i.e., $h_{\mathrm{QTL}}^{2}=\sigma_{\mathrm{QTL}}^{2} / \sigma_{P}^{2}$ ). The genotypic values derived from the final QTL model were used to compute additive allele effects (da Silva Pereira et al. 2020a).

Data availability. The phenotypic data analyzed for this study can be found as Supplementary Material. The genotypic data and linkage map information are available at https://github.com/mmollina/B2721_ map (da Silva Pereira et al. 2020b). MAPpoly (https://github.com/ mmollina/mappoly) and QTLpoly (https:/github.com/guilherme-pereira/ QTLpoly) source codes are available at their respective GitHub pages.

\section{Results}

The reaction to common scab in the B2721 mapping population was evaluated across three environments, and the raw phenotypic data, as well as the adjusted means and weights, are made available in Supplementary Table S1.

All three evaluated traits showed broad phenotypic value ranges, with several full-sib clones showing transgressive segregation, i.e., being more resistant or more susceptible when compared with parental means (Table 1). Although similar ranges were observed for all evaluated traits across environments, reaction to common scab in PA11 and ME12 was skewed toward more severe phenotypes, whereas MN11 behaved in the opposite direction, hence toward resistance (Fig. 1A to C). In fact, the mapping population mean of percentage of scabby tubers (PS) was only $18.88 \%$ in MN11, but 91.64 and $81.15 \%$ in PA11 and ME12, respectively (Table 1). Similar trends were observed for area index (AI), with 0.07 in MN11, but 0.27 and 0.31 in PA11 and ME12, and lesion index (LI), with 0.13 in MN11, but 0.60 and 0.64 in PA11 and ME12. The common check, B1829-5, also performed very inconsistently across locations, showing 95.22 and $85.00 \%$ of scabby tubers in PA11 and ME12, respectively, but only $3.85 \%$ in MN11. Variation for these traits in MN11 
could not be attributed to genetics, as evidenced by their null heritability estimates. In PA11 and ME12, the heritability values were 0.53 and 0.72 for PS, 0.48 and 0.70 for AI, and 0.78 and 0.79 for LI, respectively. For the joint model, the respective heritability values for PS, AI, and LI were $0.48,0.44$, and 0.67 (Table 1).

The correlation among separately adjusted means for different environments can be observed in Fig. 1D to F. Between PA11 and ME12 means, the correlations were positive and moderate for PS (0.42), AI (0.38), and LI (0.68), but low between these environments and MN11 ( $~ 0.05$ to 0.20$)$. The lack of consistency in the individual ranking across environments can be visualized in Fig. 1A to C, especially in relation to $\mathrm{MN11}$, implying strong genotype-by-environment interaction. The correlation estimates between the jointly adjusted means with the PA11 and ME12 means were high ( 0.77 to 0.90$)$, and with MN11 were moderate $(\sim 0.34$ to 0.40$)$. As these three environments were under natural common scab pressure, meaning that no inoculum was artificially applied to the clones, we believe that in MN11 certain environmental conditions, including the availability of less pathogenic Streptomyces spp. or strains, did not allow the disease to progress as much as in PA11 and ME12. Although MN11 had little to offer for genetic analysis purposes, the adjusted means derived from this environment were carried along with the analysis as a sort of negative control for QTL mapping.

We also compared the correlation among traits using their jointly adjusted means (Fig. 2). We observed that the traits were highly, positively correlated $(\sim 0.76$ to 0.83$)$, similar to what has been found by Zorrilla et al. (2021). This is likely related to the progression of the disease, such that in a susceptible genotype, more scabby tubers resulted in broader areas covered by scab-like lesions, which, in turn, appeared to be more severe. Despite the high correlation, we were able to

Table 1. Summary of percentage of scabby tubers (PS), area index (AI), and lesion index (LI) of common scab reaction in the B2721 potato mapping population based on separate (MN11, PA11, ME12) and joint analyses

\begin{tabular}{|c|c|c|c|c|c|c|c|}
\hline \multirow[b]{2}{*}{ Trait } & \multirow[b]{2}{*}{ Location } & \multicolumn{3}{|c|}{ Means } & \multirow[b]{2}{*}{$F_{1}$ range } & \multirow[b]{2}{*}{$\sigma_{G}^{2 \mathrm{a}}$} & \multirow[b]{2}{*}{$H^{2 \mathrm{~b}}$} \\
\hline & & 'Atlantic' & B1829-5 & $\mathbf{F}_{1}$ & & & \\
\hline \multirow[t]{4}{*}{ PS } & Joint & 72.32 & 65.12 & 63.68 & $16.13-78.40$ & $5.61 e+01$ & 0.485 \\
\hline & MN11 & $\mathrm{NA}^{\mathrm{c}}$ & 3.85 & 18.88 & $0.00-86.93$ & $4.32 e-05$ & 0.000 \\
\hline & PA11 & 100.00 & 95.22 & 91.64 & $53.57-100.00$ & $4.90 e+01$ & 0.531 \\
\hline & ME12 & NA & 85.00 & 81.15 & $0.00-100.00$ & $2.65 e+02$ & 0.724 \\
\hline \multirow[t]{4}{*}{ AI } & Joint & 0.29 & 0.24 & 0.22 & $0.02-0.44$ & $2.64 e-03$ & 0.437 \\
\hline & MN11 & NA & 0.01 & 0.07 & $0.00-0.49$ & $8.11 e-10$ & 0.000 \\
\hline & PA11 & 0.33 & 0.28 & 0.27 & $0.09-0.50$ & $3.19 e-03$ & 0.481 \\
\hline & ME12 & NA & 0.40 & 0.31 & $0.00-0.64$ & $1.48 e-02$ & 0.702 \\
\hline \multirow[t]{4}{*}{ LI } & Joint & 0.64 & 0.54 & 0.45 & $0.02-0.77$ & $1.87 e-02$ & 0.668 \\
\hline & MN11 & NA & 0.01 & 0.13 & $0.00-0.74$ & $2.60 e-09$ & 0.000 \\
\hline & PA11 & 0.79 & 0.80 & 0.60 & $0.11-1.00$ & $3.67 e-02$ & 0.777 \\
\hline & ME12 & NA & 0.72 & 0.64 & $0.00-0.98$ & $3.70 e-02$ & 0.791 \\
\hline
\end{tabular}

${ }^{\mathrm{a}} \sigma_{G}^{2}=$ genetic variance.

${ }^{\mathrm{b}} H^{2}=$ broad-sense heritability.

${ }^{\mathrm{c}} \mathrm{NA}=$ not available

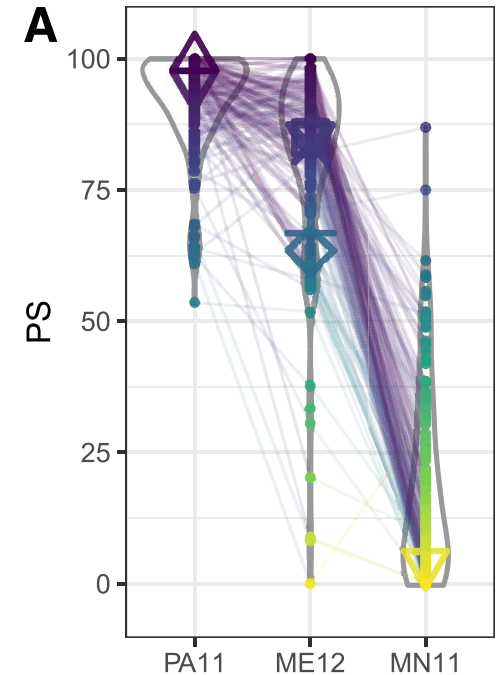

D

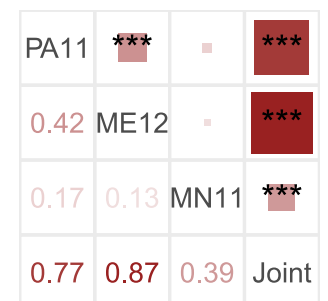

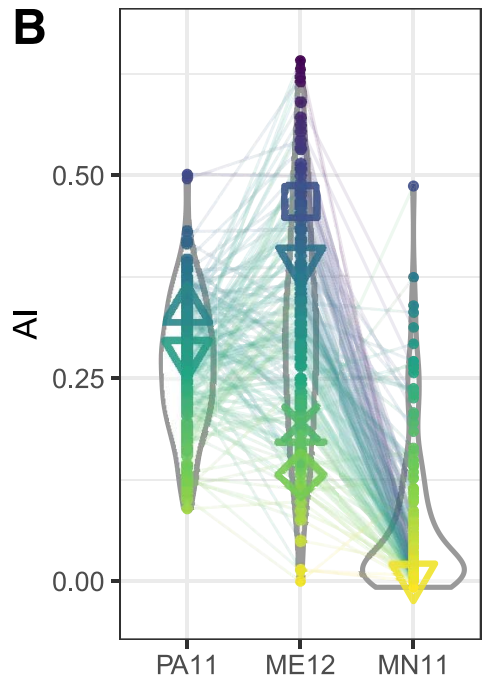

$\mathbf{E}$

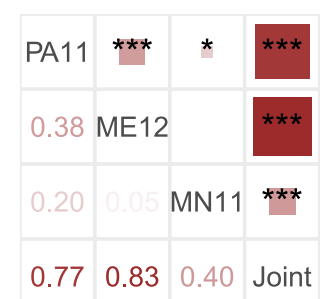

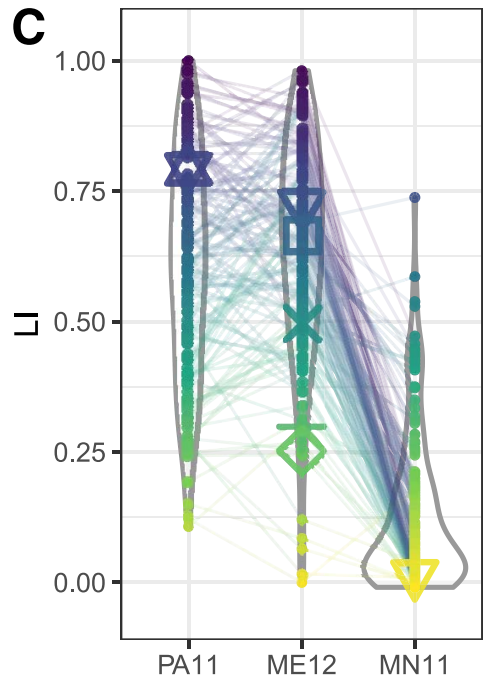

Clone

$\triangle$ Atlantic

$\square$ GreenMt

1 Ontario

X RBurbank

S Superior

$\nabla$ B1829-5

- B2721

$\mathbf{F}$

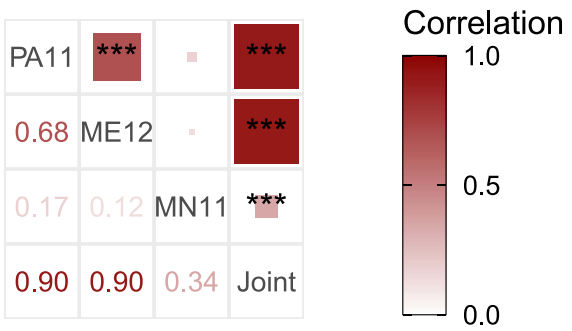

Fig. 1. A to C, Violin and D to F, correlogram plots for percentage of A and D, scabby tubers (PS), B and E, area index (Al), and C and F, lesion index (LI) of common scab reaction in the B2721 potato mapping population evaluated across three environments (MN11, PA11, ME12). Symbols represent different checks. Lines connect the same B2721 clone in consecutive environments. Correlograms show Pearson's correlation values $(* P<0.05$; $* * * P<0.001)$ between separately and jointly adjusted means. 
identify QTL only for LI. QTL mapping models were fitted using separately and jointly adjusted means derived from the phenotypic analyses. The QTL for LI was colocalized on linkage group 3 at $99 \mathrm{cM}$ for PA11, ME12, and jointly adjusted means, but no QTL was found for MN11 (Fig. 3A). This QTL explained as much as $30.1 \%$ of the trait variation in PA11. For ME12 and jointly adjusted means, the QTL heritability was 22.5 and $25.4 \%$, respectively (Table 2 ). The significance of the score statistics ( $P$ values) ranged from $4.17 e-05$ (ME12) to $1.05 e-06$ (PA11).

The QTL allele contributions for LI were relatively consistent among PA11, ME12, and joint analyses (Fig. 3B). The parent B1829-5 showed more pronounced additive effects, with allele $e$ contributing to increasing $(+0.0583)$ and allele $f$ contributing to decreasing $(-0.0476)$ the mean $(0.587) \mathrm{LI}$ in PA11. In this case, in addition to allele

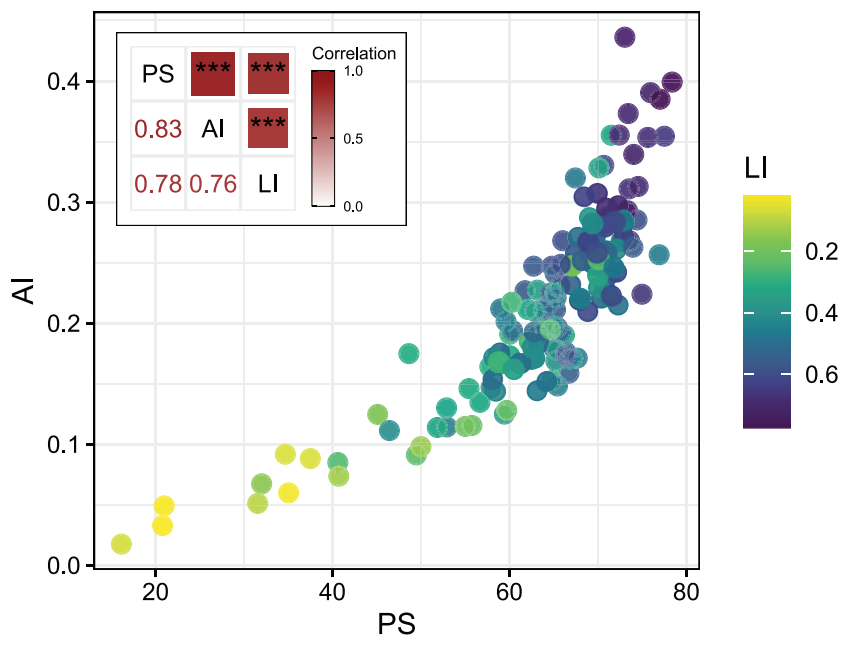

Fig. 2. Scatterplot of jointly adjusted means for percentage of scabby tubers (PS), area index (Al), and lesion index (LI) of common scab reaction in the B2721 potato mapping population evaluated across three environments. Correlogram (upper left) shows Pearson's correlation values $(* * * P<0.001)$. $f$, one would have to select allele $g$ from B1829-5, and alleles $a$ and $c$ from 'Atlantic' in order to potentially drive LI down.

The SNP markers at 95\% support interval boundaries of the QTL in PA11 ( 96 to $103 \mathrm{cM})$ were solcap_snp_c2_1830 (ST4.03ch3: 51731168) and solcap_snp_c1_7076 (ST4.03ch3:54368685). This region spanned $2,637,517 \mathrm{bp}(4.23 \%)$ of the chromosome 3 of the S. tuberosum v. 4.03 reference genome (Sharma et al. 2013) and contained 263 genes (Supplementary Table S2). The closest markers on the left and on the right of the QTL peak were solcap_snp_ c2_57263 (ST4.03ch3:53439319) and solcap_snp_c1_5812 (ST04.03ch3: 53665337), respectively, and spanned $226,018 \mathrm{bp}$; this region contained 23 genes.

\section{Discussion}

While both B2721 parents and most of the full sibs are highly susceptible to common scab for two environments, namely PA11 and ME12, the absence of more severe symptoms in MN11 can be attributed to a strong genotype-by-environment interaction. The variation observed in this environment is hence because of nongenetic, environmental effects. For all traits, the lack of correlation between MN11 and the other two environments confirms such a divergent pattern. This also explains how there was no evidence for QTL in the same region as for PA11 and ME12, where common scab was relatively more severe. That is, in order to map QTL for resistance to a disease, the environment pathogen pressure should be such that the genotypes can express their genetic merit and be phenotypically evaluated. In the case of potato common scab, the variation present within pathogenic Streptomyces spp., and other soil variables, such as moisture content and $\mathrm{pH}$, are known to influence the severity of scab (Braun et al. 2017b). The distribution of different Streptomyces spp. isolates predominating in different parts of the United States (Wanner 2009) could partially explain the lack of agreement between MN and either ME or PA.

Bradshaw et al. (2008), working with 227 full-sib progenies, encountered similar issues when only one of three environments showed scorable severity for common scab, for which heritability was 0.66. While studying 23 tetraploid potatoes, Haynes et al. (1997) found higher broad-sense heritability values for AI (0.89) and LI (0.93), where a rather low genotype-by-environment interaction was observed. For 370 clones evaluated over 9 years, Enciso-
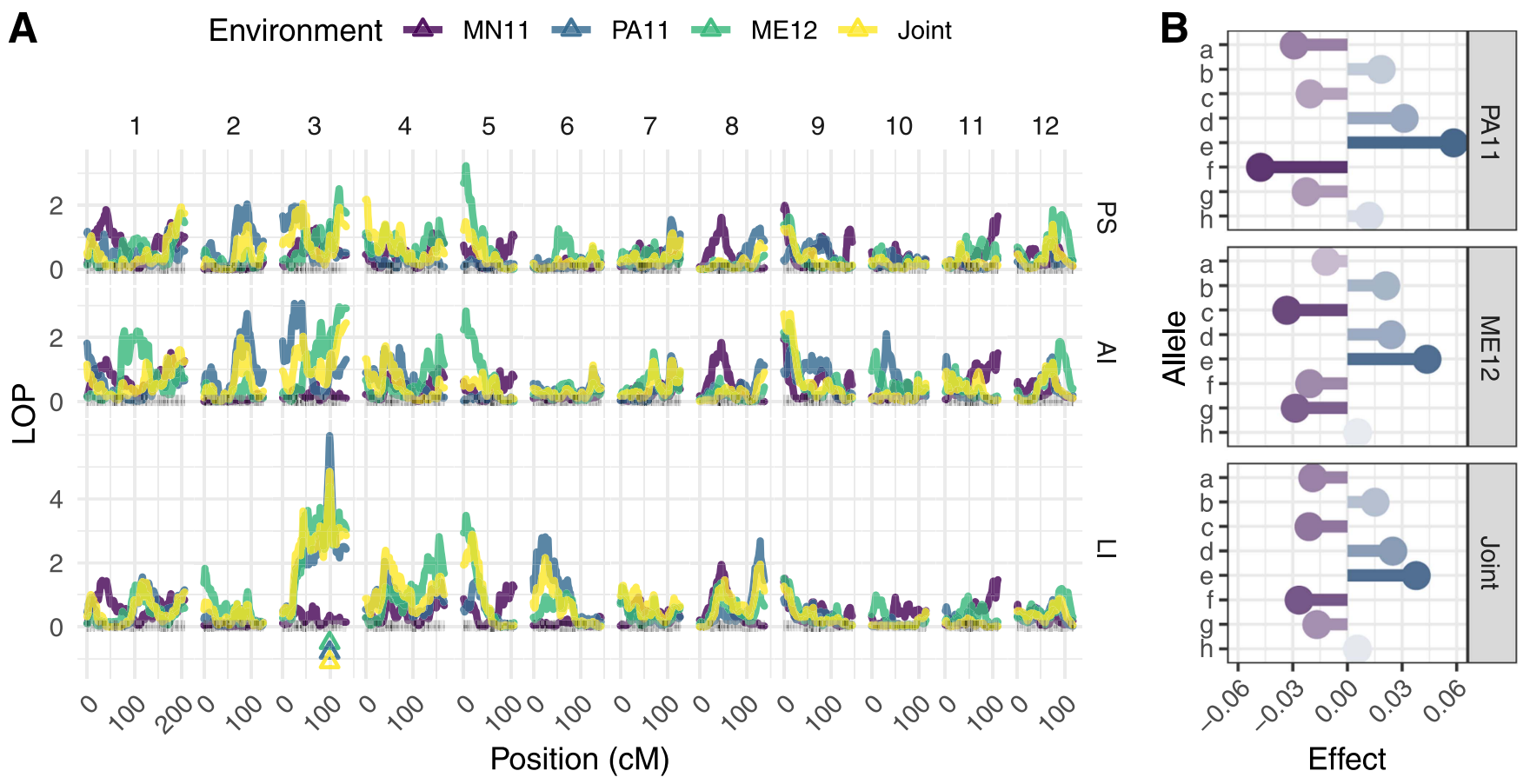

Fig. 3. A, QTL profiles for percentage of scabby tubers (PS), area index (AI), and lesion index (LI) of common scab reaction in the B2721 potato mapping population evaluated across three environments (MN11, PA11, ME12). "Joint" refers to the QTL analysis using the jointly adjusted means, triangles represent the QTL peak, and LOP = $-\log _{10}(P)$. B, Additive effects of the QTL on linkage group 3 at $99 \mathrm{cM}$ for LI in PA11, ME12, and joint analyses. Parental alleles (haplotypes): 'Atlantic' = a, b, c, d, and $B 1829-5=e, f, g, h$. 
Table 2. QTL detected for lesion index (LI) of common scab reaction in the B2721 potato mapping population based on separate (PA11, ME12) and joint analyses

\begin{tabular}{|c|c|c|c|c|c|c|c|c|}
\hline Environment & $\mathbf{L G}^{\mathbf{a}}$ & Position (cM) & $\mathrm{SI}^{\mathrm{b}}(\mathrm{cM})$ & Score & $P$ value & Intercept & $\sigma_{\mathrm{QTL}}^{2} \mathrm{c}$ & $h_{\mathrm{QTL}}^{2}{ }^{\mathrm{d}}$ \\
\hline PA11 & 3 & 99 & $96-103$ & 195.32 & $1.05 e-06$ & 0.587 & 0.0162 & 0.301 \\
\hline ME12 & 3 & 99 & $43-134$ & 131.90 & $4.17 e-05$ & 0.626 & 0.0110 & 0.225 \\
\hline Joint & 3 & 99 & $43-103$ & 161.17 & $1.40 e-05$ & 0.446 & 0.0078 & 0.254 \\
\hline
\end{tabular}

${ }^{a} \mathrm{LG}=$ linkage group.

b $\mathrm{SI}=\sim 95 \%$ support interval.

${ }^{\mathrm{c}} \sigma_{\mathrm{OTL}}^{2}=$ variance component associated with the QTL.

${ }^{\mathrm{d}} h_{\mathrm{QTL}}^{2}=$ QTL heritability.

Rodriguez et al. (2018) found low genotype-by-year interaction and a genomic heritability estimate of 0.45 for common scab scoring. In another study involving diploid potatoes, where lesion type and percentage of surface area was scored for common scab over 3 years, Braun et al. (2017b) found heritability values ranging from 0.48 to 0.79 . Finally, in a diversity panel with 148 clones, heritability was estimated as 0.81 for 3-year evaluation data (Yuan et al. 2019). Therefore, except for the null heritabilities resulting from MN11, our estimates ( $\sim 0.44$ to 0.79$)$ were in relative agreement with those found in the literature.

Some genomic regions have been found to be associated with common scab resistance in previous studies, but only one has shown evidence for a QTL on chromosome 3 as identified in the present study. Zorrilla et al. (2021) evaluated both incidence and severity of common scab under high disease pressure in a full-sib population $\left(N=151 \mathrm{~F}_{1}\right.$ clones) derived from 'Atlantic' $\times$ 'Superior' cross, with linkage maps built separately for each parent. A QTL on chromosome 3 was detected for both traits in the 'Atlantic' linkage map only. For incidence, the QTL peak on chromosome $3(84.5 \mathrm{cM})$ explained $13.3 \%$ of the total variation, and it was flanked by solcap_snp_c2_20069 (ST4.03ch3:48471111) and solcap_snp_c1_6318 (ST4.03ch3: 48856757), with support intervals $(\sim 65.5$ to $96.5 \mathrm{cM})$ ranging from solcap_snp_c2_55465 (ST4.03ch3:44517478) to solcap_snp_c2_ 18271 (ST4.03ch3:55234747). For severity, the peak on chromosome $3(92.5 \mathrm{cM})$ explained $19.4 \%$ of the total variation, and it was located between solcap_snp_c2_1724 (ST4.03ch3:51404231) and solcap_ snp_c2_1835 (ST4.03 ch3:51091650), with support intervals $(\sim 67.5$ to $95.5 \mathrm{cM}$ ) ranging from solcap_snp_c1_8059 (ST4.03ch3:45686390) to solcap_snp_c2_47843 (ST4.03ch3:54141894). These support intervals overlap those of our QTL on chromosome 3 ( $\sim 51.73$ to $54.37 \mathrm{Mbp}$ ). Interestingly, two alleles of 'Atlantic' (named 1 and 4) were found to decrease incidence and severity in their progeny (Zorrilla et al. 2021), similar to what alleles $a$ and $c$ did in our B2721 population (Fig. 3B).

In our study, LI was more suitable for QTL detection, likely because of its consistently higher broad-sense heritability (0.777 at PA11, 0.791 at ME12) in relation to those from $\mathrm{AI}(0.481$ at PA11, 0.702 at ME12), combined with a broader range in LI $(0.11$ to 1.00 at PA11, $\sim 0$ to 0.98 at ME12) when compared with that of $\mathrm{AI}(0.09$ to 0.50 at PA11, $\sim 0$ to 0.64 at ME12) (Fig. 1; Table 1). B1829-5 was relatively less susceptible than 'Atlantic', but both parents showed alleles contributing to either increasing or decreasing LI (Fig. 3B). The individual allele effects were relatively small (ranging from -0.048 for allele $f$ up to +0.058 for allele $e$ in PA11), but it is the combination of four alleles (two from each parent) that determines the departure from the mean (0.587 at PA11). Note that the fact that allele effects were different within each parent leading to a segregating $F_{1}$ contributes to the QTL detection (da Silva Pereira et al. 2020b).

Interestingly, Kaiser et al. (2020) used association (GWAS) instead of linkage analysis to evaluate a mapping population $\left(N=198 \mathrm{~F}_{1}\right.$ clones) derived from the 'Tundra' $\times$ 'Kalkaska' cross. In this case, only dosage, which is less informative than haplotypes, was taken into consideration when searching for QTL. Modernly, molecular breeding has been taking advantage of the high-density markers covering the whole genome to perform selection based on genomic estimated breeding values. For potato common scab, such genomic-assisted prediction models have shown prediction accuracies as high as 0.278 , and an SNP with a major effect on chromosome 9 (Enciso-Rodriguez et al. 2018). Haplotypic and
QTL information, such as that provided here, can be used to leverage genomic-assisted prediction models in order to deliver higher predictive abilities, notably for less complex traits (Gemenet et al. 2020). However, high-throughput genotyping is not the only factor that matters in such studies. In fact, both population size and phenotyping accuracy are important experimental factors that impact QTL detection and reliable allele effect estimation. Therefore, in the case of such a complicated trait, one might want to try larger population sizes (e.g., $N>200 \mathrm{~F}_{1}$ clones). Larger plots, field nurseries, or glasshouses tests under artificial infection are potentially other factors that need to be taken into consideration when carrying out disease-related QTL studies.

At least, 3 of the 23 genes located within the QTL marker interval were previously implicated in plant responses to biotic stresses related to signaling pathways or hypersensitive responses, namely MYB transcription factor (PGSC0003DMT400063172, 53.60 Mbp) (Ambawat et al. 2013), calcium-dependent protein kinase 1 (CDPK1; PGSC0003DMT400034291, 53.43 Mbp) (Lee and Rudd 2002), and ubiquitin-protein ligase (PGSC0003DMT400092368, 53.59 Mbp) (Craig et al. 2009; Duplan and Rivas 2014). In addition, there were several other genes among the remaining 240 transcripts retrieved from the $95 \%$ QTL support interval that were known for encoding proteins involved in plant defense, such as receptor-like kinase (PGSC00 03DMT400046807, 52.47 Mbp; PGSC0003DMT400046778, 52.57 Mbp) (Nazarian-Firouzabadi et al. 2019), proteins containing nucleotide biding-ARC domain (PGSC0003DMT400087730, 52.37 Mbp) and leucine-rich repeat (LRR; PGSC0003DMT400046655, 52.18 Mbp) (Takken et al. 2006), and transcription factors such as NAC (PGSC 0003DMT400097372, 54.15 Mbp; PGSC0003DMT400096000, $\sim 54.17 \mathrm{Mbp}$ ) (Nuruzzaman et al. 2013) and WRKY (PGSC0003DMT 400046570, 53.04) (Bhattarai et al. 2010; Enciso-Rodriguez et al. 2018).

Although the list of candidate genes is still hypothetical, several transcripts encoding related protein isoforms (e.g., MYB, WRKY, LRR receptor-like serine/threonine-protein kinase) were found to be differentially expressed between the resistant 'Hindenburg' and susceptible 'Green Mountain' inoculated with Streptomyces scabies (Fofana et al. 2020), and the resistant 'Kalkaska' and the susceptible 'Tundra' treated with Thaxtomin A (Kaiser et al. 2020). Here, we detected a single QTL consistently in two of three environments, which explains up to $30 \%$ of the variation for lesion index. In order to effectively apply genes and haplotypes in breeding, the QTL identified in this study needs to be further investigated in a more diverse genetic background. If an oligogenic-like inheritance confirms and QTL are validated, specific markers to retrieve the haplotypes conferring resistance to common scab can be screened in breeding populations to perform early selection (Bradshaw 2017). Progress of MAS in potato is relatively slow when compared with inbred, diploid species, and needs to take into consideration several aspects in addition to the genetic architecture of a trait, such as polyploidy, high heterozygosity, and clonal propagation (Bethke et al. 2019; Slater et al. 2014).

\section{Acknowledgment}

C. Thill passed away in 2014. This paper is dedicated to his memory.

\section{Literature Cited}

Ambawat, S., Sharma, P., Yadav, N. R., and Yadav, R. C. 2013. MYB transcription factor genes as regulators for plant responses: an overview. Physiol. Mol Biol. Plants 19:307-321. 
Bethke, P. C., Halterman, D. A., and Jansky, S. H. 2019. Potato germplasm enhancement enters the genomics era. Agronomy (Basel) 9:575.

Bhattarai, K. K., Atamian, H. S., Kaloshian, I., and Eulgem, T. 2010. WRKY72type transcription factors contribute to basal immunity in tomato and Arabidopsis as well as gene-for-gene resistance mediated by the tomato $\mathrm{R}$ gene Mi-1. Plant J. 63:229-240.

Bradshaw, J. E. 2017. Review and analysis of limitations in ways to improve conventional potato breeding. Potato Res. 60:171-193.

Bradshaw, J. E., Hackett, C. A., Pande, B., Waugh, R., and Bryan, G. J. 2008. QTL mapping of yield, agronomic and quality traits in tetraploid potato (Solanum tuberosum subsp. tuberosum). Theor. Appl. Genet. 116:193-211.

Braun, S. R., Endelman, J. B., Haynes, K. G., and Jansky, S. H. 2017a. Quantitative trait loci for resistance to common scab and cold-induced sweetening in diploid potato. Plant Genome 10:1-9.

Braun, S. R., Gevens, A., Charkowski, A., Allen, C., and Jansky, S. 2017b. Potato common scab: a review of the causal pathogens, management practices, varietal resistance screening methods, and host resistance. Am. J. Potato Res. 94: 283-296.

Butler, D. G., Cullis, B. R., Gilmour, A. R., Gogel, B. J., and Thompson, R. 2018. ASReml-R Reference Manual Version 4. Hemel Hempstead. http:// www.homepages.ed.ac.uk/iwhite/asreml/uop

Cai, J. 2019. ggcorrplot2: visualize a correlation matrix using ggplot2 v. 0.1.0. https://github.com/caijun/ggcorrplot2

Chen, J., Zhang, F., Wang, L., Leach, L., and Luo, Z. 2018. Orthogonal contrast based models for quantitative genetic analysis in autotetraploid species. New Phytol. 220:332-346.

Clarke, C. R., Kramer, C. G., Kotha, R. R., Wanner, L. A., Luthria, D. L., and Kramer, M. 2019. Cultivar resistance to common scab disease of potato is dependent on the pathogen species. Phytopathology 109:1544-1554.

Craig, A., Ewan, R., Mesmar, J., Gudipati, V., and Sadanandom, A. 2009. E3 ubiquitin ligases and plant innate immunity. J. Exp. Bot. 60:1123-1132.

Cullis, B. R., Smith, A. B., and Coombes, N. E. 2006. On the design of early generation variety trials with correlated data. J. Agric. Biol. Environ. Stat. 11:381-393.

da Silva Pereira, G., Gemenet, D. C., Mollinari, M., Olukolu, B. A., Wood, J. C., Diaz, F., Mosquera, V., Gruneberg, W. J., Khan, A., Buell, C. R., Yencho, G. C., and Zeng, Z.-B. 2020a. Multiple QTL mapping in autopolyploids: a random-effect model approach with application in a hexaploid sweetpotato full-sib population. Genetics 215:579-595.

da Silva Pereira, G., Mollinari, M., Schumann, M. J., Clough, M. E., and Yencho, C. 2020b. The recombination landscape and multiple QTL mapping in a Solanum tuberosum cv. 'Atlantic'-derived F1 population. Heredity 126: $817-830$.

Dees, M. W., and Wanner, L. A. 2012. In search of better management of potato common scab. Potato Res. 55:249-268.

Duplan, V., and Rivas, S. 2014. E3 ubiquitin-ligases and their target proteins during the regulation of plant innate immunity. Front. Plant Sci. 5:42

Enciso-Rodriguez, F., Douches, D., Lopez-Cruz, M., Coombs, J., and de los Campos, G. 2018. Genomic selection for late blight and common scab resistance in tetraploid potato (Solanum tuberosum). G3 8:2471-2481.

Felcher, K. J., Coombs, J. J., Massa, A. N., Hansey, C. N., Hamilton, J. P., Veilleux, R. E., Buell, C. R., Douches, D. S. 2012. Integration of two diploid potato linkage maps with the potato genome sequence. PLoS One 7: e36347.

Fofana, B., Somalraju, A., Fillmore, S., Zaidi, M., Main, D., and Ghose, K. 2020. Comparative transcriptome expression analysis in susceptible and resistant potato (Solanum tuberosum) cultivars to common scab (Streptomyces scabies) revealed immune priming responses in the incompatible interaction. PLoS One 15:e0235018.

Gemenet, D. C., Lindqvist-Kreuze, H., De Boeck, B., da Silva Pereira, G., Mollinari, M., Zeng, Z.-B., Yencho, G. C., and Campos, H. 2020. Sequencing depth and genotype quality: accuracy and breeding operation considerations for genomic selection applications in autopolyploid crops. Theor. Appl. Genet. 133:3345-3363.

Goth, R. W., Haynes, K. G., and Wilson, D. R. 1993. Evaluation and characterization of advanced potato breeding clones for resistance to scab by cluster analysis. Plant Dis. 77:911-914.

Hackett, C. A., Bradshaw, J. E., and Bryan, G. J. 2014. QTL mapping in autotetraploids using SNP dosage information. Theor. Appl. Genet. 127: 1885-1904.

Harrel, Jr., F. E., 2019. Hmisc: Harrell Miscellaneous v. 4.3-0. https://cran.r-project.org/web/packages/Hmisc/index.html

Haynes, K. G., Christ, B. J., Burkhart, C. R., and Vinyard, B. T. 2009. Heritability of resistance to common scab in diploid potatoes. Am. J. Potato Res. 86:165-170.

Haynes, K. G., Goth, R. W., and Young, R. J. 1997. Genotype x environment interactions for resistance to common scab in tetraploid potato. Crop Sci. 37: 1163-1167.

Haynes, K. G., Wanner, L. A., Thill, C. A., Bradeen, J. M., Miller, J., Novy, R. G., Whitworth, J. L., Corsini, D. L., and Vinyard, B. T. 2010. Common scab trials of potato varieties and advanced selections at three U.S. locations. Am. J. Potato Res. 87:261-276.

Horsfall, J. G., and Barratt, R. W. 1945. An improved grading system for measuring plant diseases. Phytopathology 35:655.
Isik, F., Holland, J., and Maltecca, C. 2017. Genetic Data Analysis for Plant and Animal Breeding. Springer, Berlin.

James, C. 1971. A Manual of Assessment Keys for Plant Diseases. American Phytopathological Society, St. Paul, MN.

Kaiser, N. R., Coombs, J. J., Felcher, K. J., Hammerschmidt, R., Zuehlke, M. L., Buell, C. R., and Douches, D. S. 2020. Genome-wide association analysis of common scab resistance and expression profiling of tubers in response to thaxtomin A treatment underscore the complexity of common scab resistance in tetraploid potato. Am. J. Potato Res. 97:513-522.

Kinkel, L. L., Bowers, J. H., Shimizu, K., Neeno-Eckwall, E. C., and Schottel, J. L. 1998. Quantitative relationships among thaxtomin A production, potato scab severity, and fatty acid composition in Streptomyces. Can. J. Microbiol. 44:768-776.

Krištůfek, V., Diviš, J., Omelka, M., Kopecký, J., and Sagová-Marečková, M. 2015. Site, year and cultivar effects on relationships between periderm nutrient contents and common scab severity. Am. J. Potato Res. 92:473-482.

Lee, J., and Rudd, J. J. 2002. Calcium-dependent protein kinases: versatile plant signalling components necessary for pathogen defence. Trends Plant Sci. 7:97-98

Loria, R., Bukhalid, R. A., Creath, R. A., Leiner, R. H., Olivier, M., and Steffens, J. C. 1995. Differential production of thaxtomins by pathogenic streptomyces species in vitro. Phytopathology 85:537-541.

McCord, P. H., Sosinski, B. R., Haynes, K. G., Clough, M. E., and Yencho, G. C. 2011. Linkage mapping and QTL analysis of agronomic traits in tetraploid potato (Solanum tuberosum subsp. tuberosum). Crop Sci. 51:771-785.

Möhring, J., and Piepho, H. P. 2009. Comparison of weighting in two-stage analysis of plant breeding trials. Crop Sci. 49:1977-1988.

Mollinari, M., and Garcia, A. A. F. 2019. Linkage analysis and haplotype phasing in experimental autopolyploid populations with high ploidy level using hidden Markov models. G3 9:3297-3314.

Mollinari, M., Olukolu, B. A., da Silva Pereira, G., Khan, A., Gemenet, D., Yencho, G. C., and Zeng, Z.-B. 2020. Unraveling the hexaploid sweetpotato inheritance using ultra-dense multilocus mapping. G3 10:281-292.

Murphy, A. M., De Jong, H., and Tai, G. C. C. 1995. Transmission of resistance to common scab from the diploid to the tetraploid level via $4 \mathrm{x}-2 \mathrm{x}$ crosses in potatoes. Euphytica 82:227-233.

Navarro, F. M., Rak, K. T., Banks, E., Bowen, B. D., Higgins, C., and Palta, J. P. 2015. Strategies for selecting stable common scab resistant clones in a potato breeding program. Am. J. Potato Res. 92:326-338.

Nazarian-Firouzabadi, F., Joshi, S., Xue, H., and Kushalappa, A. C. 2019 Genome-wide in silico identification of LysM-RLK genes in potato (Solanum tuberosum L.). Mol. Biol. Rep. 46:5005-5017.

Nuruzzaman, M., Sharoni, A. M., and Kikuchi, S. 2013. Roles of NAC transcription factors in the regulation of biotic and abiotic stress responses in plants. Front. Microbiol. 4:248.

Rosyara, U. R., De Jong, W. S., Douches, D. S., and Endelman, J. B. 2016 Software for genome-wide association studies in autopolyploids and its application to potato. Plant Genome 9:1-10.

Schmitz Carley, C. A., Coombs, J. J., Douches, D. S., Bethke, P. C., Palta, J. P., Novy, R. G., and Endelman, J. B. 2017. Automated tetraploid genotype calling by hierarchical clustering. Theor. Appl. Genet. 130:717-726.

Schumann, M. J., Zeng, Z. B., Clough, M. E., and Yencho, G. C. 2017. Linkage map construction and QTL analysis for internal heat necrosis in autotetraploid potato. Theor. Appl. Genet. 130:2045-2056

Sharma, S. K., Bolser, D., de Boer, J., Sonderkaer, M., Amoros, W., Carboni, M. F., et al. 2013. Construction of reference chromosome-scale pseudomolecules for potato: integrating the potato genome with genetic and physical maps. G3 3:2031-2047.

Slater, A. T., Cogan, N. O. I., Hayes, B. J., Schultz, L., Dale, M. F. B. Bryan, G. J., and Forster, J. W. 2014. Improving breeding efficiency in potato using molecular and quantitative genetics. Theor. Appl. Genet. 127:2279-2292.

Sverrisdóttir, E., Byrne, S., Sundmark, E. H. R., Johnsen, H. Ø., Kirk, H. G. Asp, T., Janss, L., and Nielsen, K. L. 2017. Genomic prediction of starch content and chipping quality in tetraploid potato using genotyping-by-sequencing Theor. Appl. Genet. 130:2091-2108.

Takken, F. L., Albrecht, M., and Tameling, W. I. L. 2006. Resistance proteins: molecular switches of plant defence. Curr. Opin. Plant Biol. 9:383 390

Uitdewilligen, J. G. A. M. L., Wolters, A.-M. A., D'hoop, B. B., Borm, T. J. A. Visser, R. G. F., and van Eck, H. J. 2013. A next-generation sequencing method for genotyping-by-sequencing of highly heterozygous autotetraploid potato. PLoS One 8:e62355.

Vos, P. G., Uitdewilligen, J. G. A. M. L., Voorrips, R. E., Visser, R. G. F., and van Eck, H. J. 2015. Development and analysis of a 20K SNP array for potato (Solanum tuberosum): an insight into the breeding history. Theor. Appl. Genet. 128:2387-2401

Wanner, L. A. 2006. A survey of genetic variation in Streptomyces isolates causing potato common scab in the United States. Phytopathology 96: 1363-1371. 
Wanner, L. A. 2009. A patchwork of Streptomyces species isolated from potato common scab lesions in North America. Am. J. Potato Res. 86:247-264.

Wanner, L. A., and Haynes, K. G. 2009. Aggressiveness of Streptomyces on four potato cultivars and implications for common scab resistance breeding. Am. J. Potato Res. 86:335-346.

Wickham, H. 2016. ggplot2: Elegant Graphics for Data Analysis, 2nd ed. Springer, New York.

Yuan, J., Bizimungu, B., De Koeyer, D., Rosyara, U., Wen, Z., and Lagüe, M. 2019. Genome-wide association study of resistance to potato common scab. Potato Res. 63:253-266.
Zorrilla, C., Navarro, F., Vega-Semorile, S., and Palta, J. 2021. QTL for pitted scab, hollow heart and tuber calcium identified in a tetraploid population of potato derived from Atlantic $\times$ Superior cross. Crop Sci. 61: 1630-1651.

Zou, F., Fine, J. P., Hu, J., and Lin, D. Y. 2004. An efficient resampling method for assessing genome-wide statistical significance in mapping quantitative trait loci. Genetics 168:2307-2316.

Zych, K., Gort, G., Maliepaard, C. A., Jansen, R. C., and Voorrips, R. E. 2019. FitTetra 2.0 - improved genotype calling for tetraploids with multiple population and parental data support. BMC Bioinformatics 20:148. 